\title{
Calculate Central Limit Theorem for the Number of Empty Cells after Allocation of Particles
}

\author{
Odila Abduraimovna Islamova \\ Tashkent University of Information Technologies, Uzbekistan \\ Corresponding author email: odilova-islamova@mail.ru \\ Zoya Sergeevna Chay \\ Tashkent University of Information Technologies, Uzbekistan \\ Email: chay1526@mail.ru \\ Feruza Saidovna Rakhimova \\ Tashkent University of Information Technologies, Uzbekistan \\ Email:feruzatuit@mail.ru \\ Feruza Saydaxmatovna Abdullayeva \\ Tashkent University of Information Technologies, Uzbekistan \\ Email: abdullayevaferuza@gmail.com
}

\begin{abstract}
This work belongs to the field of limit theorems for separable statistics. In particular, this paper considers the number of empty cells after placing particles in a finite number of cells, where each particle is placed in a polynomial scheme. The statistics under consideration belong to the class of separable statistics, which were previously considered in (Mirakhmedov: 1985), where necessary statements for the layout of particles in a countable number of cells were proved. The same scheme was considered in (Asimov: 1982), in which the conditions for the asymptotic normality of random variables were established. In this paper, the asymptotic normality of the statistics in question is proved and an estimate of the remainder term in the central limit theorem is obtained. In summary, the demand for separable statistics systems is growing day by day to address large-scale databases or to facilitate user access to data management. Because such systems are not only used for data entry and storage, they also describe their structure: file collection supports logical consistency; provides data processing language; restores data after various interruptions; database management systems allow multiple users.
\end{abstract}

Keywords---central limit theorem, particle placement, particle, polynomial scheme, separable statistics

\section{Introduction}

This paper considers a scheme of random placement of particles in cells. The same scheme was considered in Mirakhmedov (1988); Kolchin et al. (1976), in which the conditions of asymptotic normality were established for the number of cells containing $r$ particles. In Asimov (1982), in the arrangement of two types of particles, threedimensional symmetric separable statistics PC $\left(\mu_{00}, \sum_{r \geq 0} \mu_{0 r}, \sum_{r \geq 0} \mu_{r 0}\right)$ was studied, where $\mu_{r_{1} r_{2}}$ - are the number of cells containing $r_{I}$ particles of the first and $r_{2}$ particles of the second type, respectively. In the present work, we studied the distribution of particles of s types in a finite number of cells (Mirakhmedov, 1987; Mirakhmedov, 1989; Popova, 1968). The considered value is $\mu_{0}$ - the number of empty cells after placing all $s$ types of particles in which each type of particles is placed in a polynomial scheme (Mitchell et al., 2006; Valjarević \& Petrović, 2020; 
Indahyati \& Sintaasih, 2019; Benedicta, 2021). The asymptotic normality of statistics $\mu_{0}$ is proved, and an estimate of the remainder term in the central limit theorem is obtained (Ivchenko \& Levin, 1978; Mikhailov, 1981).

\section{Main Results}

Let s types of particles be allocated independently of each other and consistently in $N$ cells, the number of $l$-type particles be equal to $n_{l}$, each of them falls into the cell with the number $m$ with the probability (Hall, 1984; Dedecker \& Rio, 2000).

$$
P_{l m}>0, \quad m=\overline{1 ; N}, P_{l 1}+\ldots+P_{l N}=1, l=\overline{1 ; s}
$$

Consider the following random variable $\mu_{o}(s)$ - the number of empty cells after the allocation of all $n_{1}, \ldots, n_{s}$ particles. Evidently, each type of particles is allocated by the polynomial scheme, i.e. the random vector.

$$
\eta_{l}=\left(\eta_{l 1}, \ldots, \eta_{l N}\right)
$$

Can be set by the conditional distribution of the random vector $\xi_{l}=\left(\xi_{l 1}, \ldots, \xi_{l N}\right)$, where we have:

$$
Z\left(\xi_{l m}\right)=\prod\left(n_{l} p_{l m}\right), m=\overline{1 ; N}, l=\overline{1 ; s},
$$

For independent random variables $\xi_{l m}$

$$
\begin{aligned}
& \text { Set } \xi_{l}^{(s)}=\left(\xi_{l 1}, \ldots, \xi_{l N}\right), \lambda_{l m}=n_{l} P_{l m}, \\
& \alpha_{l}=n_{l} /{ }_{N}, \lambda_{m}=\lambda_{1 m}+\ldots+\lambda_{s m}, \\
& A_{n}(s)=\sum_{m=1}^{N} e^{-\lambda_{m}}, \gamma_{e}=\frac{1}{n_{l}} \sum_{m=1}^{N} \lambda_{l m} e^{-\lambda_{m}} \\
& \sigma_{N}^{2}(s)=\sum_{m=1}^{N} e^{-\lambda_{m}}\left[1-e^{-\lambda_{m}}-\sum_{m=1}^{N} \alpha_{l} \gamma_{l}^{2}\right]
\end{aligned}
$$

We suppose that for each $l=1, \ldots, s$

$$
\max N_{p_{l m}} \leq C_{\circ} \quad, \quad \ln \alpha_{l} \leq \varepsilon N
$$

Theorem. There exists $C(s)$ such that

$$
\Delta_{N}^{(s)}(y)=\left|P\left(\frac{\mu_{0}(s)-A_{n}(s)}{\sigma_{N}(s)}<y\right)-\Phi(y)\right| \leq C(s)\left[\sigma_{N}^{-1}(s)+\sum_{m=1}^{N}\left(n_{m}\right)^{-1}\right]
$$


Proof

We consider the random variable:

$$
g\left(\xi_{m}^{(s)}\right)=f\left(\xi_{m}^{(s)}\right)-e^{-\lambda_{m}}+\sum_{l=1}^{s} \gamma_{l}\left(\xi_{l m}-\lambda_{l m}\right),
$$

Where $f\left(\xi_{m}^{(s)}\right)=f\left(\xi_{l 1}, \ldots, \xi_{l s}\right)$ - a random function of non-conventional integer arguments.

According to the work we have:

$$
\Delta_{N}^{(s)}(y) \leq C(s)\left[\frac{1}{\sigma_{N}^{3}(s)} \sum_{m=1}^{N} E\left|g\left(\xi_{m}^{(s)}\right)\right|^{3}+\sum_{j=1}^{s} \frac{1}{n_{j}}\right]
$$

We will cush the value $\sigma_{N}^{2}(s)$ in the form:

$$
\sigma_{N}^{2}(s)=\sum_{m=1}^{N}\left(1-\left(1+\lambda_{m}\right) e^{-\lambda_{m}}\right) e^{-\lambda_{m}}+\sum_{m=1}^{N} \sum_{l=1}^{S} \lambda_{l m}\left(e^{\lambda_{m}}-\gamma_{l}\right)^{2}
$$

And the random value $g\left(\xi_{m}^{(s)}\right)$ we write in the form:

$$
g\left(\xi_{m}^{(s)}\right)=f\left(\xi_{m}^{(s)}\right)+e^{-\lambda_{m}} \sum_{e=1}^{S} \xi_{l m}-\left(1+\lambda_{m}\right) e^{-\lambda_{m}}+\sum_{e=1}^{S}\left(\gamma_{l}-\lambda_{m}\right)\left(\xi_{l m}-\lambda_{l m}\right)
$$

Then:

$$
\begin{gathered}
E\left|g\left(\xi_{m}^{(s)}\right)\right|^{3} \leq 4 E\left|f\left(\xi_{m}^{(s)}\right)+e^{-\lambda_{m}} \sum_{j=1}^{s} \xi_{j m}-\left(1+\lambda_{m}\right) e^{-\lambda_{m}}\right|^{3}+ \\
+4 s^{2} \sum_{j=1}^{s}\left|\gamma_{j}-e^{-\lambda_{m}}\right|^{3} E\left|\xi_{j m}-\lambda_{j m}\right|^{3}=4 \Delta_{1 m}+4 s^{2} \Delta_{2 m}
\end{gathered}
$$

The random variable $\xi_{1 m}+\ldots .+\xi_{s m}$ has Pousson distribution with parameter $\lambda_{m}$. Moreover, the distribution of random variable $f\left(\xi_{m}^{(s)}\right)$ coincides with distribution of random variable $f\left(\xi_{1 m}+\ldots .+\xi_{s m}\right)$, where:

$$
f(0)=1 \text { and } f(y)=0 \text {, if } y>0 \text {. }
$$

Thus

$$
\begin{aligned}
\Delta_{1 m} & =E\left|f\left(\xi_{1 m}+\ldots+\xi_{s m}\right)+e^{-\lambda_{m}}\left(\xi_{1 m}+\ldots+\xi_{s m}\right)-\left(1+\lambda_{m}\right) e^{-\lambda_{m}}\right|^{3}= \\
& =e^{-\lambda_{m}}\left(1-e^{-\lambda_{m}}\left(1+\lambda_{m}\right)\right)^{3}+\lambda_{m}^{4} e^{-4 \lambda_{m}}+ \\
& +\sum_{j=2}^{\infty} \mid j-1-\lambda_{m} e^{-4 \lambda_{m}} \cdot \frac{\lambda_{m}^{j}}{j !}=\Delta_{1 m}^{\prime}+\Delta_{1 m}^{\prime \prime}+\Delta_{1 m}^{\prime \prime \prime}
\end{aligned}
$$

Since $(1+u) e^{-u} \leq 1$ for $u>0$, then 


$$
\sum_{m=1}^{N} \Delta_{1 m}^{\prime} \leq \sum_{m=1}^{N} e^{-\lambda_{m}}\left(1-e^{-\lambda_{m}}\left(1+\lambda_{m}\right)\right) \leq \sigma_{N}^{2}(s)
$$

From the fact that $u^{2} e^{-2 u} \leq 1$ and $\frac{1}{2} u^{2} e^{-u} \leq 1-(1+u) e^{-u} \quad$ we receive:

$$
\sum_{m=1}^{N} \Delta_{1 m}^{\prime \prime} \leq \sum_{m=1}^{N} \lambda_{m}^{2} e^{-2 \lambda_{m}} \leq 2 \sum_{m=1}^{N}\left(1-\left(1+\lambda_{m}\right) e^{-\lambda_{m}}\right) \leq 2 \sigma_{N}^{2}(s)
$$

Let $\sum_{\lambda_{m} \leq 1}, \sum_{\lambda_{m}>1}$ means summation by those $m$, for which $\lambda_{m} \leq 1$ and $\lambda_{m}>1$ respectively. We have:

$$
\sum_{\lambda_{m \leq 1}} \Delta_{1 m}^{\prime \prime \prime}=\sum_{\lambda_{m \leq 1}} e^{-\lambda_{m}^{3}}\left[E\left(\xi_{1 m}+\ldots+\xi_{s m}-1-\lambda_{m}\right)^{3}-\left(1+\lambda_{m}\right)^{3} e^{-\lambda_{m}^{3}}+\lambda_{m}^{4} e^{-\lambda_{m}}\right]
$$

Let $\xi$ Poisson random variable with parameter. Then for any $l \geq 2$

$$
E(\xi-\lambda)^{l}=\sum \frac{l ! \lambda_{m}^{k_{2}+\ldots+k_{l}}}{k_{2} ! \ldots k_{l} !(2 !)^{k_{2}} \ldots(l !)^{k_{l}}}
$$

In $\sum^{1}$ sum over all nonnegative integers $k_{2}, \ldots, k_{l}$ such that $2 k_{2}+\ldots+l k_{l}=l$.

So:

$$
E|\xi-\lambda|^{l}=c(l)\left(\lambda^{i / 2}+\lambda\right)^{l / l^{\prime}}
$$

Where:

$$
l^{\prime}=\left\{\begin{aligned}
l, & \text { if } \quad l=2 k \\
l-1, & \text { if } \quad l=2 k+1 \quad, k \in z
\end{aligned}\right.
$$

Hense for $\lambda_{m} \leq 1$ we have: $E\left|\xi_{1 m}+\ldots+\xi_{s m}-1-\lambda_{m}\right|^{3} \leq c\left(\lambda_{m}+1\right) \leq c$

Therefore, given that when $\lambda_{m} \leq 1$

$$
\begin{gathered}
\left(1+\lambda_{m}\right)^{3}=1+3 \lambda_{m}+3 \lambda^{2}+\lambda^{3} \leq 1+3 \lambda_{m}+C 3 \lambda_{m}^{2}, \text { then similarly (5) we have: } \\
\sum_{\lambda_{m} \leq 1} \Delta_{{ }^{\prime \prime}}^{\prime \prime} \leq c \sum_{m=1}^{N} \lambda_{m}^{2} e^{-2 \lambda_{m}} \leq c \sigma_{N}^{2}(s)
\end{gathered}
$$

Further due to (6) and (7) and inequalities between moments we set: 


$$
\begin{aligned}
& \sum_{\lambda_{m}>1} \Delta_{1 m}^{\prime \prime \prime} \leq \sum_{\lambda_{m}>1} e^{-3 \lambda_{m}} E\left|\xi_{1 m}+\ldots+\xi_{s m}-\lambda_{m}-1\right|^{3} \leq \\
& \leq \sum_{\lambda_{m}>1}\left[E\left(\xi_{1 m}+\ldots+\xi_{s m}-\lambda_{m}-1\right)^{4}\right]^{3 / 4 \cdot e^{-3 \lambda_{m}}} \leq \\
& \leq c \sum_{\lambda_{m}>1} \lambda_{m}^{3 / 2} e^{-3 \lambda_{m}} \leq c \sum_{\lambda_{m}>1} \lambda_{m}^{2} e^{-2 \lambda_{m}^{2}} \leq c \sigma_{N}^{2}(s)
\end{aligned}
$$

Summing up the ratios (1)-(9) we receive:

$$
\sum_{m=1}^{N} \Delta_{1 m} \leq C \delta_{N}^{2}(s)(10)
$$

Estimate the value $\sum_{m=1}^{N} \Delta_{2 m}$. Since $\lambda_{j m} \leq C_{0} \alpha_{j}$, that for $\alpha_{j} \leq 1$, receive $\lambda_{j m} \leq C_{0}$.

Given this ration we have:

$$
\begin{aligned}
& \sum_{m=1}^{N}\left|\gamma_{j}-e^{-\lambda_{m} \mid}\right|^{3} E\left|\xi_{j m}-\lambda_{j m}\right|^{3} \leq C \sum_{\lambda_{j m} \leq 1}\left(\gamma_{j}-e^{-\lambda_{m}}\right)^{3} \sum_{l=1}^{\infty}\left|i-\lambda_{j m}\right|^{3} \pi_{i}\left(\lambda_{i m}\right)+ \\
& +\sum_{\lambda_{j m}>1}\left(\gamma_{j}-e^{-\lambda_{m}}\right)^{3}\left[E\left|\xi_{j m}-\lambda_{j m}\right|^{4}\right]^{3 / 4} \leq \\
& \leq C\left[\sum_{\lambda_{j m} \leq 1}\left(\gamma_{j}-e^{-\lambda_{m}}\right)^{2}\left(E\left(\xi_{j m}-\lambda_{j m}\right)^{3}+2 \lambda_{j m}^{3} e^{-\lambda_{j m}}\right)+\right. \\
& \left.+\sum_{\lambda_{j m}>1}\left(\gamma_{j}-e^{-\lambda_{m}}\right)^{2} \lambda_{m}^{3 / 2}+\sum_{\lambda_{j m}>1}\left|\gamma_{j}-e^{-\lambda_{m}}\right|^{3} \lambda_{j m}^{3 / 2}\right] \leq \\
& \leq c \sigma_{N}^{2}(s)+\sum_{\lambda_{j m}>1}\left|\gamma_{j}-e^{-\lambda_{m}}\right|^{3} \cdot \lambda_{j m}^{3 / 2}
\end{aligned}
$$

When obtaining the last inequality, the relations were taken into account (6) and (7).

Let:

$$
\lambda_{j m}>1, \lambda_{j}>1 \text {. Then } \gamma_{j}<1 / \alpha_{j} .
$$

Therefore:

$$
\left|\gamma_{j}-e^{-\lambda_{m}}\right| \lambda_{m}^{1 / 2} \leq \gamma_{j} \lambda_{m}^{1 / 2}+1 \leq \frac{\sqrt{c_{0}}}{\sqrt{\alpha_{j}}}+1 \leq \sqrt{c_{0}}+1
$$

Hence:

$$
\begin{aligned}
& \sum_{\lambda_{j m}>1}\left[\left|\gamma_{j}-e^{-\lambda_{m}}\right| \lambda_{m}^{1 / 2}\right]^{3} \leq\left(\sqrt{c_{0}}+1\right) \sum_{m=1}^{N}\left(\gamma_{j}-e^{-\lambda_{m}}\right)^{2} \lambda_{j m} \leq \\
& \leq\left(\sqrt{c_{0}}+1\right) \sigma_{N}^{2}(s)
\end{aligned}
$$


From here and from (11) receive:

$$
\sum_{m=1}^{N} \Delta_{2 m} \leq c \sigma_{N}^{2}(s)
$$

This from (1), (10), (12) we have:

$$
\sum_{m=1}^{N} E\left|g\left(\xi_{m}^{(s)}\right)\right|^{3} \leq c(s) \sigma_{N}^{2}(s)
$$

From (1) and (13) follows the theorem. Theorem is proved.

\section{Conclusions}

In summary, the demand for separable statistics systems is growing day by day to address large-scale databases or to facilitate user access to data management (El-Zonkoly, 2011; Lee et al., 2015; Chaloupka et al., 1974; Ardell, 1972). Because such systems are not only used for data entry and storage, they also describe their structure: file collection supports logical consistency; provides data processing language; restores data after various interruptions; database management systems allow multiple users to work in parallel (Degond et al., 1999; Cesati \& Trevisan, 1997). The main requirement for considers the number of empty cells after placing particles in a finite number of cells, where each particle is placed in a polynomial scheme. Systems is to safely store external data and to respond to the request of the user to satisfy it. This requires the completeness of the information stored in the medical database to maintain the integrity of the data (Rakhimov et al., 2021). If the GPU is the Device, then the size of the partition and blocks is limited. Each block is executed on a separate multiprocessor independently of other blocks. Therefore, the width and height of the block are determined by the maximum number of simultaneously processed threads on the multiprocessor. In turn, when executed, the blocks are divided into bundles of 32 threads, which are launched in accordance with the commands of the multiprocessor thread control unit. Communication between threads in a block is done using shared memory and barrier thread synchronization (Rakhimov et al., 2021).

\section{References}

Ardell, A. J. (1972). The effect of volume fraction on particle coarsening: theoretical considerations. Acta metallurgica, 20(1), 61-71. https://doi.org/10.1016/0001-6160(72)90114-9

Asimov, I. (1982). Exploring the earth and the cosmos: the growth and future of human knowledge. New York: Crown.

Benedicta, O. (2021). Relationship between competitive intelligence and competitive advantage in manufacturing industry. International Research Journal of Management, IT and Social Sciences, 8(5), 342-351. https://doi.org/10.21744/irjmis.v8n5.1908

Cesati, M., \& Trevisan, L. (1997). On the efficiency of polynomial time approximation schemes. Information Processing Letters, 64(4), 165-171. https://doi.org/10.1016/S0020-0190(97)00164-6

Chaloupka, V., Barbaro-Galtieri, A., Chew, D. M., Kelly, R. L., Lasinski, T. A., Rittenberg, A., ... \& Particle Data Group. (1974). Review of particle properties. Physics Letters B,50(1), i. https://doi.org/10.1016/03702693(74)90738-2

Dedecker, J., \& Rio, E. (2000, January). On the functional central limit theorem for stationary processes. In Annales de l'Institut Henri Poincare (B) Probability and Statistics (Vol. 36, No. 1, pp. 1-34). No longer published by Elsevier. https://doi.org/10.1016/S0246-0203(00)00111-4

Degond, P., Peyrard, P. F., Russo, G., \& Villedieu, P. (1999). Polynomial upwind schemes for hyperbolic systems. Comptes Rendus de l'Académie des Sciences-Series I-Mathematics, 328(6), 479-483. https://doi.org/10.1016/S0764-4442(99)80194-3

El-Zonkoly, A. M. (2011). Optimal placement of multi-distributed generation units including different load models using particle swarm optimization. Swarm and Evolutionary Computation, 1(1), 50-59. https://doi.org/10.1016/j.swevo.2011.02.003

Hall, P. (1984). Central limit theorem for integrated square error of multivariate nonparametric density estimators. Journal of multivariate analysis, 14(1), 1-16. https://doi.org/10.1016/0047-259X(84)90044-7 
Indahyati, N., \& Sintaasih, D. K. (2019). The relationship between organizational justice with job satisfaction and organizational citizenship behavior. International Research Journal of Management, IT and Social Sciences, 6(2), 63-71. https://doi.org/10.21744/irjmis.v6n2.611

Ivchenko, G. I., \& Levin, V. V. (1978). Asymptotic normality in the scheme of simple random sampling without replacement. Theory of Probability \& Its Applications, 23(1), 93-105.

Kolchin, V. F., Sevastianov, B. A., \& Chistyakov, V. P. (1976). Random accommodations. Science, Moscow.

Lee, C. S., Ayala, H. V. H., \& dos Santos Coelho, L. (2015). Capacitor placement of distribution systems using particle swarm optimization approaches. International Journal of Electrical Power \& Energy Systems, 64, 839851. https://doi.org/10.1016/j.ijepes.2014.07.069

Mikhailov, V. G. (1981). The central limit theorem for a scheme of independent allocation of particles by cells. Trudy Matematicheskogo Instituta imeni VA Steklova, 157, 138-152.

Mirakhmedov, S. A. (1987). Estimates for the convergence rate in the central limit theorem for randomized separable statistics in a multinomial scheme. Journal of Soviet Mathematics, 38(6), 2346-2357.

Mirakhmedov, S. A. (1988). Approximation of the Distribution of Multidimensional Randomized Divisible Statistics by Normal Distributions (Multinomial Scheme). Theory of Probability \& Its Applications, 32(4), 696-706.

Mirakhmedov, S. A. (1989). Randomized decomposable statistics in a generalized allocation scheme over a countable set of cells. Diskretnaya matematika, 1(4), 46-62.

Mitchell, M. W., Genton, M. G., \& Gumpertz, M. L. (2006). A likelihood ratio test for separability of covariances. Journal of Multivariate Analysis, 97(5), 1025-1043. https://doi.org/10.1016/j.jmva.2005.07.005

Popova, T. Y. (1968). Limit theorems in a model of distribution of particles of two types. Theory of Probability \& Its Applications, 13(3), 511-516.

Rakhimov, B. S., Mekhmanov, M. S., \& Bekchanov, B. G. (2021, April). Parallel algorithms for the creation of medical database. In Journal of Physics: Conference Series (Vol. 1889, No. 2, p. 022090). IOP Publishing.

Rakhimov, B., Rakhimova, F., Sobirova, S., \& Allaberganov, O. (2021). Mathematical Bases Of Parallel Algorithms For The Creation Of Medical Databases. InterConf.

Valjarević, D., \& Petrović, L. (2020). Statistical causality and separable processes. Statistics \& Probability Letters, 167, 108915. https://doi.org/10.1016/j.spl.2020.108915 